\title{
Cognitive Issues in the Perception of Desktop Virtual Environments
}

\author{
Robert D. Macredie and Sonali S. Morar \\ Department of Information Systems and Computing \\ Brunel University, Uxbridge, Middlesex UB8 3PH, UK ${ }^{\dagger}$
}

\begin{abstract}
Desktop virtual environments are computer-generated representations of three-dimensional spaces, such as buildings, displayed on a normal desktop monitor. They are used to convey physical world representations in an interactive manner, which makes them ideal for modeling and training applications. The accuracy of the representations is very important as they impact on the way in which users interact with the environment. This paper presents a review of the cognitive issues involved with one key area related to representation and subsequent interaction, the visual perception of the desktop virtual environments. The overall aim of the review is to formulate an agenda for research, which would contribute, through basic empirical investigations, to the further understanding of visually perceiving desktop virtual environments. The review concentrates on visual cues because it is essentially these visual cues that integrate to provide the illusion of depth and the third dimension. The review begins by highlighting the importance of the research through assessing the use of the desktop virtual environment tool in various commercial settings. It then progresses to consider the perceptual theories that underpin perceiving visual cues and theories relating to visual cue combination and conflict. The final aspect of the review attempts to emphasize the idea of individual differences of visual cue perception in the desktop virtual environment context. Individual differences consider cognitive and perceptual capabilities, which is a growing concern in the pursuit of conducive desktop virtual environment displays.
\end{abstract}

${ }^{\dagger}\{$ Robert.Macredie\}, \{Sonali.Morar\}@brunel.ac.uk 


\section{INTRODUCTION}

Desktop Virtual Environments present three-dimensional interactive graphics on the computer screen. These graphics can be considered a more accessible form of virtual reality because they do not require the use of extra viewing equipment, such as head mounted displays. As a result, these graphics attempt to provide a sense of dimensionality without the use of stereoscopic visual cues and still remain popularly used within commercial industries, such as the computer games industry. Such popularity highlights the importance of understanding how individuals visually comprehend desktop virtual environments and effectively accomplish their specific tasks. The EPSRC (2000) has acknowledged that further research should concentrate on the fundamentals of virtual reality to highlight, through basic empirical investigations, the essential attributes of a desktop virtual environment display. The attributes considered within this paper are visual depth cues and their effects when perceiving desktop virtual environments.

Visual depth cues integrate to create the sense of depth that occurs stereoscopically when perceiving the real world. As desktop virtual environment displays are monocular in nature, visual depth cues, such as luminance, texture, and relative height, play an important role in creating the dimensionality. Extensive research has been undertaken across the broad range of visual depth cues to investigate their effects when perceiving (Dosher et al., 1986, Wickens et al., 1989, Hendrix \& Barfield 1995, Surdick et al., 1997, Westerman \& Cribbin, 1998). The implications of many earlier studies attempted to understand visual perception in the real world (Koffka, 1935, Hebb, 1949, Gibson, 1950, Weintraub \& Walker, 1973, Cutting, 1986), and the implications of recent visual depth cues studies in the virtual reality community attempt to understand the perception of immersive virtual environment systems. Such systems present two disparate images to each eye to create the stereoscopic cues that are used when perceiving depth in the real world (Hendrix \& Barfield, 1995, Surdick et al., 1997). Therefore, although literature exists in the area of visual depth cues, little has been revealed about their effects when perceiving desktop virtual environments.

In this paper, to contribute further understanding of the use of visual depth cues when perceiving desktop virtual environments, we aim to present 
an agenda for research. The agenda is threefold:

1. The first stage considers visual depth cues in isolation and identifies the individual strengths of each and the effects of specific combinations when visually perceiving the desktop virtual presentations. The objective is to provide a comprehensive review of how the visual depth cues contribute to effective desktop virtual environments and to consider the results broadly with respect to the cue combination theories that describe the various strategies that are used by individuals when perceiving combinations of visual cues at one time.

2. The second stage examines visual cue conflict, which refers to the ability of certain visual cues to provide conflicting depth information about a particular desktop virtual environment display. This aspect is especially important to understand because desktop virtual environments are interactive and dynamic and often used to replicate real-world situations, such as training applications in the aviation industry.

3. The third and final area extends the visual cue review to consider individual differences in visual cue use when perceiving desktop virtual environments. The term 'individual differences' refers to the effects of the individual's cognitive and perceptual abilities when conducting interactive, visual search tasks. This topic has been covered quite extensively as a specific research field (Carroll 1993, Dillon \& Watson, 1996, Chen et al., 2000) and can be applied to the area of visual depth cues to answer questions about the types of visual cues that individuals employ to perceive desktop virtual environments.

To highlight the importance of the area, the paper begins by looking at the types of industries using desktop virtual environments and then provides the relevant background to visual perception and visual depth cues before proposing the research agenda.

\section{DESKTOP VIRTUAL ENVIRONMENTS}

This section provides an overview of various industries using desktop virtual environments: the commercial games industry, the aviation industry, the medical field, and the motor industry. The aim of the section is to illustrate the 
importance of exploring the perceptual issues of desktop virtual environments, given the extent to which it is being used in commercial industries.

- Games industry: The games industry is probably the most notable area in which desktop virtual environments have been used. Virtual Reality games are most often played by viewing stand-alone personal computers or televisions that are connected to play stations like Sega. Players navigate through the three-dimensional environments and manipulate the virtual objects to compete against virtual opponents and gain points. Players can often feel immersed within the desktop virtual environments, without the need for added equipment to be used for perceiving immersive virtual environments (Csikszentmihalyi \& Kubey, 1981).

- Aviation industry: The effective presentation of the third dimension has also been a critical issue within the aviation industry. Wickens et al. (1989) produced a comprehensive report that extensively considered the attributes of a two-dimensional display of three-dimensional graphics. The importance of understanding how these graphics are perceived was evident from the underlying nature of the safety critical tasks involved. It is essential for air force personnel and flight control staff to accurately perceive the three-dimensional presentations so that they can make precise spatial judgments (Hendrix \& Barfield, 1995). Therefore, the desktop virtual environments would require careful construction to adhere to an individual's perceptual abilities. Although the virtual displays used for flight control tasks and aviation training are well designed and developed, the literature fails to provide commercial virtual environment developers with sufficient instruction regarding how they can achieve this level of accuracy in their developments (Wickens et al., 1989). Often the desktop virtual developments in the commercial industries rely on successive trial and error procedures before reaching an acceptable or even commendable standard of desktop virtual environment package (Hubbold et al., 1993). Therefore, the performance of such procedures suggests that a limited amount of basic research has been undertaken about how individuals accurately perceive desktop virtual environments.

- Medical field: The games and aviation industries have been identified as prominent users of desktop virtual environments for three-dimensional 
presentations. Desktop virtual environment graphics are used by a variety of other industrial areas, including the medical field, in which images of internal human organs are used for analysis or training. The issue within this field is to produce accurate and effective three-dimensional imagery from biological information that has been traditionally presented as twodimensional, static images (McConathy \& Doyle, 1991). Concerns extending from this would be the practitioner's ability to comprehend the three-dimensional images when they are already familiar with extracting the necessary diagnoses from the two-dimensional images, such as Magnetic Resonance Imaging (MRI) and X-rays.

- Motor industry: Once again, the issue of effectively perceiving the desktop virtual environment has been highlighted and asserted as important because of the safety critical nature of the visual tasks involved. This could also be reflected in the use of desktop virtual environments for supporting architectural and planning work. Although $\mathrm{CAD} / \mathrm{CAM}$ packages have been extensively used for engineering purposes, the growth of the virtual environment concept has expanded and developed into the current manufacturing processes (Deitz, 1995; Aleksandric \& Duboka, 1997). The motor industry has used virtual reality for designing and manufacturing processes. CAD/CAM models are extended in these particular instances to create visualizations that are more realistic. For instance, it is possible to create virtual models of a car to ensure that it meets the necessary ergonomic and safety requirements. In turn, these virtual prototypes enable the car manufacturer to see a variety of alternative representations of the car whilst reducing the costs involved with creating actual prototypes (Aleksandric \& Duboka, 1997).

From this overview of the various commercial industries that use desktop virtual environments, it is possible to see the growing market for desktop virtual environment applications; therefore, understanding how these particular two-dimensional presentations are perceived as three-dimensional scenes becomes crucial. If visually effective desktop virtual environments have been developed, then the contributing factors should be understood (Ellis et al., 1997, Stanney et al., 1998, Westerman \& Cribbin, 1998). It is therefore necessary to explore the cognitive psychological area of visual perception and 
to identify the theories that form the foundation of visual cue usage in perceiving desktop virtual environments.

\section{PERCEPTUAL ISSUES}

This section explores the various approaches underpinning the manner by which individuals perceive visual depth cues. The aim is to consider whether individuals have the innate ability to recognize visual depth cues or whether the experience and familiarity with the three-dimensional environment would contribute to perceiving visual depth cues. 'Nativist', 'empiricist', direct', and 'indirect' approaches to perception are considered. (Koffka, 1935; Hebb, 1949; Gibson, 1950; Weintraub \& Walker, 1973; Cutting, 1986; Gordon, 1989). These approaches highlight the two contrasting views of visual perceptionthe one in which perception occurs naturally and the other that relies on experience and learning to nurture perception.

\section{Nativism and Empiricism}

Visual Perception encompasses theories tending to bias toward particular viewpoints that are known as either nativism or empiricism (Gordon, 1989). Gibson (1950) summarizes nativism as the innate ability of individuals to perceive their environments. Empiricism takes the view that visual perception is a learned process that is achieved through experience. Certain theorists have acknowledged the contribution of each view toward developing their particular models of visual perception (Koffka, 1935; Gibson, 1950; Gordon, 1989; Marr, 1982). For instance, the Gestalt theorists considered perception as an innate characteristic, yet they seemed to concede to the idea that certain aspects of visual perception could be influenced by such variables as familiarity, practice, and learning (Koffka, 1935; Gordon, 1989).

An empiricist's viewpoint is emphasised by the neurophysiological theory of Hebb (1949). This theory has been described as being in contrast to the nativist view of Gestalt theorists (Weintraub \& Walker, 1973). Neurophysiological theory considers the neurological activities that may be involved with visual perception. Three characteristics have been defined by 
Hebb (1949): primitive unity, nonsensory unity, and identity. Primitive unity assumes that persons can gain only minimal visual information from their first visual experience. As their visual experiences increase, however, such persons acquire knowledge about perception, enabling them to recognize objects that have not been seen before. This phenomenon is referred to as nonsensory unity and identity. As the neurophysiological theory describes the neurological activities that may be involved with perception, it proposes to account for the general perception concepts formulated by the Gestalt psychologists (Koffka, 1935; Hebb, 1949; Weintraub \& Walker, 1973). For example, according to the Gestalt psychologists' principles of organization, individuals would instinctively perceive attributes within an environment as simple, organized configurations. The neurophysiological theory proposes, however, that individuals have learned how to recognize the configurations of environmental attributes through their general visual experiences.

\section{Direct and Indirect Approaches}

The Gestalt theory is considered a 'direct approach' to visual perception, which suggests that individuals perceive the environment directly from information that is available within the environment (Gibson, 1950; Gordon, 1989). Essentially, this direct approach is considered innate, which implies that individuals have the instinctive ability to visually perceive (Cutting, 1986). Gordon (1989) also agrees that although the Gestalt theorists may have acknowledged that perception could be influenced by such factors as familiarity, practice, and learning, they still would have maintained that the perceptual process was an inherent one (Gordon, 1989).

Gibson (1950) was also a strong advocate of the direct approach of visual perception. He believed that humans did learn how to visually perceive, but that this process was undertaken through evolution and not throughout an individual's lifetime (Gordon, 1989). Gibson's theory involves the concepts of 'invariants' and 'affordances'.

- Invariants are higher-order properties, which remain constant during change. For example, if an object were moving rapidly, then the textures of the object would remain the same at any point of the movement, and therefore the flow of textures would be invariant. 
- Affordances are the properties of an object that govern its use. For example, Gibson (1968) illustrates that an apple affords eating, grasping, and throwing.

These two concepts have fuelled years of debate, which has resulted in further and expanded studies by researchers such as Norman (1998).

The opposing 'indirect approach' considers visual perception as an inferential process (Cutting, 1986). Cutting (p. 226) explains that indirect perception involves a process of deduction and therefore "goes beyond the information given". Gordon (1989) associates the indirect approach to the empiricists' view of visual perception. Examples of empiricists who inclined toward the indirect approach include Helmhholz, Brunswik, Marr, and Gregory. The central feature of the empiricist view is that visual perception is a construction or a process involving specific steps that promote the notion of experience. For example, Marr (1982) proposed the computational theory, which takes an engineering approach to visual perception and describes a series of stages that are involved in building the visual perceptions by initially using light densities, then adding other visual cues, such as texture and motion, and finally determining the positions and orientations of the attributes.

These approaches form the basis on which to consider the way in which individuals visually understand depth cues:

- The empiricist approach seems to imply that visual depth cues are perceived and understood through learning and experience (Cutting 1986; Gordon, 1989).

- The nativist approach suggests that individuals have innate mechanisms that provide them with the ability to recognize the visual depth cues from birth (Gibson, 1950, Cutting, 1986, Gordon, 1989).

Empiricists would have had to assume that individuals have an innate ability to make inferences, and nativists would have had to rely on the idea of experiences to establish innate characteristics, which diminishes the notion of pure ideologies (Gordon, 1989). This notion is reflected in the theory proposed by Gregory (1998) - known as 'the hypothesis theory'-which involves the association of neural activities to the individual's current knowledge to produce psychological data on which hypotheses are based. The idea is that the hypotheses enable individuals to make sense of the world or to perceive their environment (Gordon, 1989). Gordon also presents various 
arguments supporting the hypothesis theory, including the ability of an individual to deduce a particular object, such as a table, even though it may be viewed as having only three legs and a triangular top. This example illustrates that perception may involve more than just sensory inputs.

\section{Picture Perception}

The hypothesis theory has been applied to perceiving pictures. Gregory (1988) discusses that although individuals may perceive the lines, surfaces, and objects in a picture, in reality the objects may be bigger. Therefore, he believes that cognition plays a significant role in the perception of pictures (Gordon, 1989, Gregory, 1998). Gibson (1950; 1954; 1971) discusses extensively the theories of picture perception and proposes a theory that assumes that pictures may be perceived using some of the visual cues that are provided in the real world. An experiment conducted by Hochberg and Brooks (1962) involved identifying whether a child of 19 months, who had been exposed to and taught only the names of real toys and other objects, could recognize the objects in a picture without having seen pictures before in its life. The results showed that the child was able to recognize the picture of an object without any help or instructions (Pizlo \& Scheesesele, 1998). This empirical example does appear to support Gibson's $(1954 ; 1971)$ picture perception theory.

The theories discussed in the previous section have provided a basis on which to understand the nature of visual cues and the manner by which they are perceived. Various approaches have been explored, such as nativist and empiricism, direct and indirect (Koffka, 1935; Hebb, 1949; Gibson, 1950; Weintraub \& Walker, 1973; Cutting, 1986; Gordon, 1989). These approaches highlight the two contrasting views of visual perception-one in which perception occurs naturally and the other that relies on experience and learning to nurture perception. It has been acknowledged that certain theories may be biased toward one approach but will also accept the potential influence of the other (Gordon, 1989). Therefore, it is useful to acknowledge that whereas certain aspects of perceiving the visual depth cues may occur instinctively, recognition of and familiarity with visual depth cues may improve through experience with desktop virtual environments. The following 
section provides an overview of certain studies that have investigated visual depth cues.

\section{VISUAL DEPTH CUES}

The importance of understanding monocular visual depth cues is emphasized through the nature of desktop virtual environments. Visual depth cues are used to create three-dimensional graphics and could be considered in the same category as pictures or paintings. The essence of desktop virtual environments is the presentation of depth and dimensionality on a twodimensional display. Therefore, understanding the manner by which the visual depth cues are perceived can be considered important (Hendrix \& Barfield, 1995; Nagata, 1991). Surdick et al. (1997) explain that the area of human depth perception requires more research, in particular the effects of manipulating depth cues and the implications on the accuracy of the resulting depth perceptions and the identification of depth cues that may provide inconsistent visual perceptions. This section attempts to present a broad review of studies that have been conducted in the area of visual depth cues. The aim is to highlight that although the literature has identified visual cues that are considered dominant or weak, the implications of the various studies are wide and varying, illustrating the need for empirical investigations of visual depth cue effects when perceiving desktop virtual environments.

\section{Visual Cue Studies}

Many studies have been undertaken on a broad range of visual depth cues (Dosher et al., 1986; Wickens et al., 1989; Hendrix \& Barfield, 1995; Surdick et al., 1997; Westerman \& Cribbin, 1998). The methods used and the contexts of their implications vary considerably. and as a result, determining how transferable or applicable the results are to the desktop virtual environment area is difficult. Reviewing the studies to establish possible bases for comparisons is still useful. Table 1 shows some of the studies that have considered various visual depth cues. This is not an exhaustive list of studies, but instead illustrates a few that can be considered notable. The table shows 
the studies in chronological order, the cues that they examined, and their results. The review is essentially an overview of visual cue results and does not take into account the methods or tasks involved in achieving the specific results (for a review that does so, see Surdick et al., 1997). The reason for this exclusion is so that both review studies and empirical studies could be broadly reviewed to ascertain the general consensus of dominant and weak visual depth cues. Dosher et al. (1986), however, have acknowledged that contextual factors can influence the visual cue outcomes.

An analysis of Table 1 will now be undertaken with respect to the categories-light, texture, occlusion, and motion-proposed by Hendrix and Barfield (1995) because acknowledging the evaluations of visual depth cue studies to establish a basis for comparisons to the desktop virtual environment context is important. The table shows that light, luminance, and relative brightness - which have been considered similar — are relatively weak visual cues (Dosher et al., 1986; Wickens et al., 1989; Surdick et al., 1997; Westerman \& Cribbin, 1998). The texture category included linear perspective, according to Hendrix and Barfield's (1995) category listing. Texture was found to be a relatively weaker visual cue, and linear perspective was considered effective when perceiving varying distances but still rather weak (Wickens et al., 1989; Hendrix \& Barfield, 1995; Surdick et al., 1997, Westerman \& Cribbin 1998). The occlusion and interposition category included relative size and height visual cues. The table shows that these cues were quite strong (Wickens et al., 1989), although motion and stereopsis (when both eyes view disparate images of the same scene and these are combined to visually capture the sense of depth) were considered as dominant cues (Dosher et al., 1986; Wickens et al., 1989). Table 1 shows that certain studies (Dosher et al., 1986; Bruno \& Cutting, 1988; Massaro, 1988; Landy et al., 1995) have investigated particular visual cues in terms of combination theories and not individual effects, therefore assessing the cues and their individual roles in perception is difficult. The results of these particular investigations have been summarized in the table and will now be expanded and discussed further.

As combinations of visual depth cues represent dimensionality within the desktop virtual environments, identifying the various models that attempt to explain the manner by which the visual combinations occur is important. The models include the Weak Observer, the Strong Observer, the Modified Weak 


\begin{tabular}{|c|c|c|c|c|c|c|c|}
\hline \multirow[t]{3}{*}{ 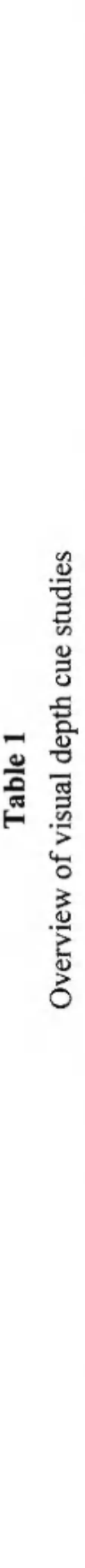 } & 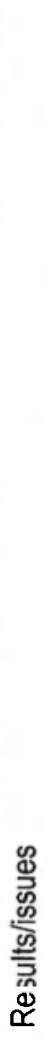 & 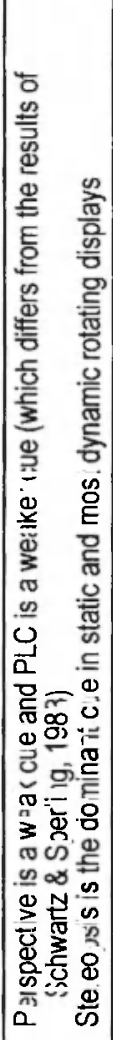 & 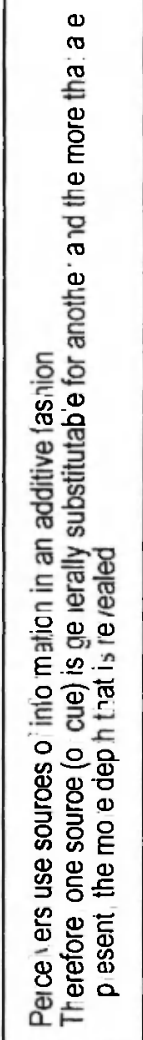 & 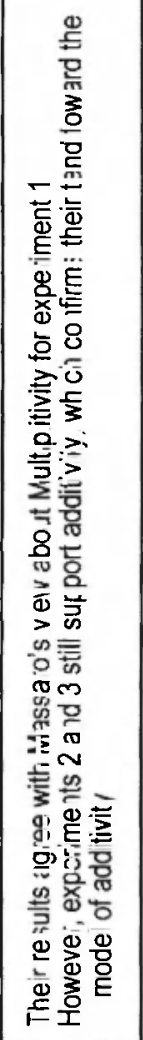 & 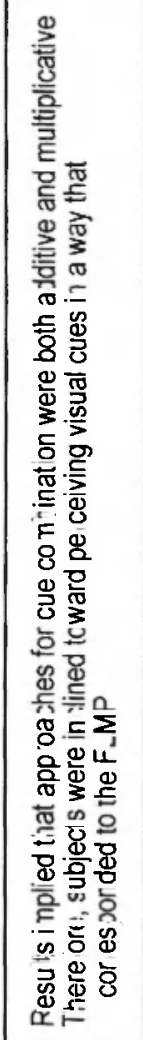 & 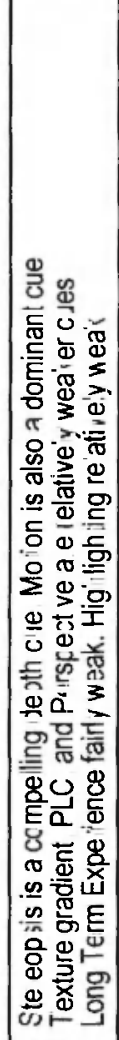 & 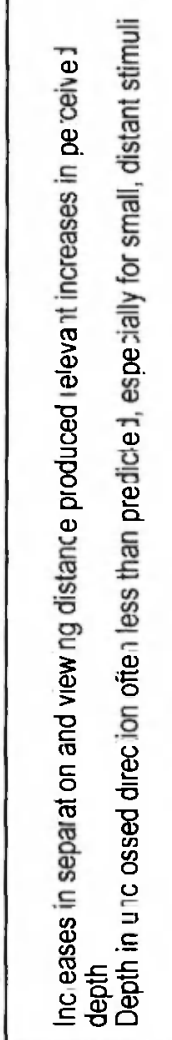 \\
\hline & 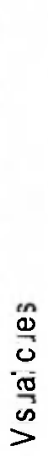 & 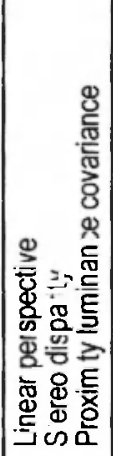 & 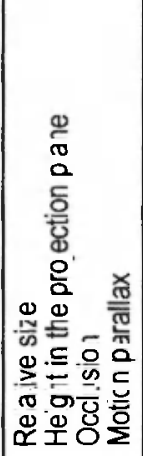 & 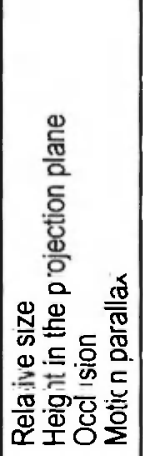 & 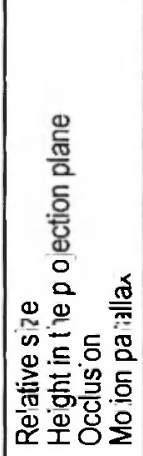 & 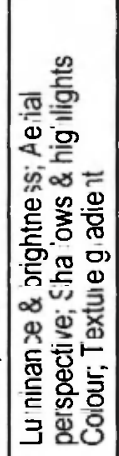 & 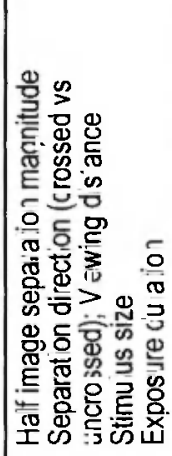 \\
\hline & $\ddot{\alpha}$ & 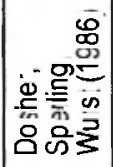 & 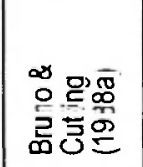 & 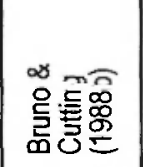 & 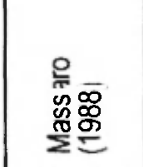 & 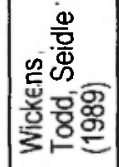 & 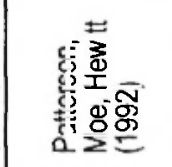 \\
\hline
\end{tabular}




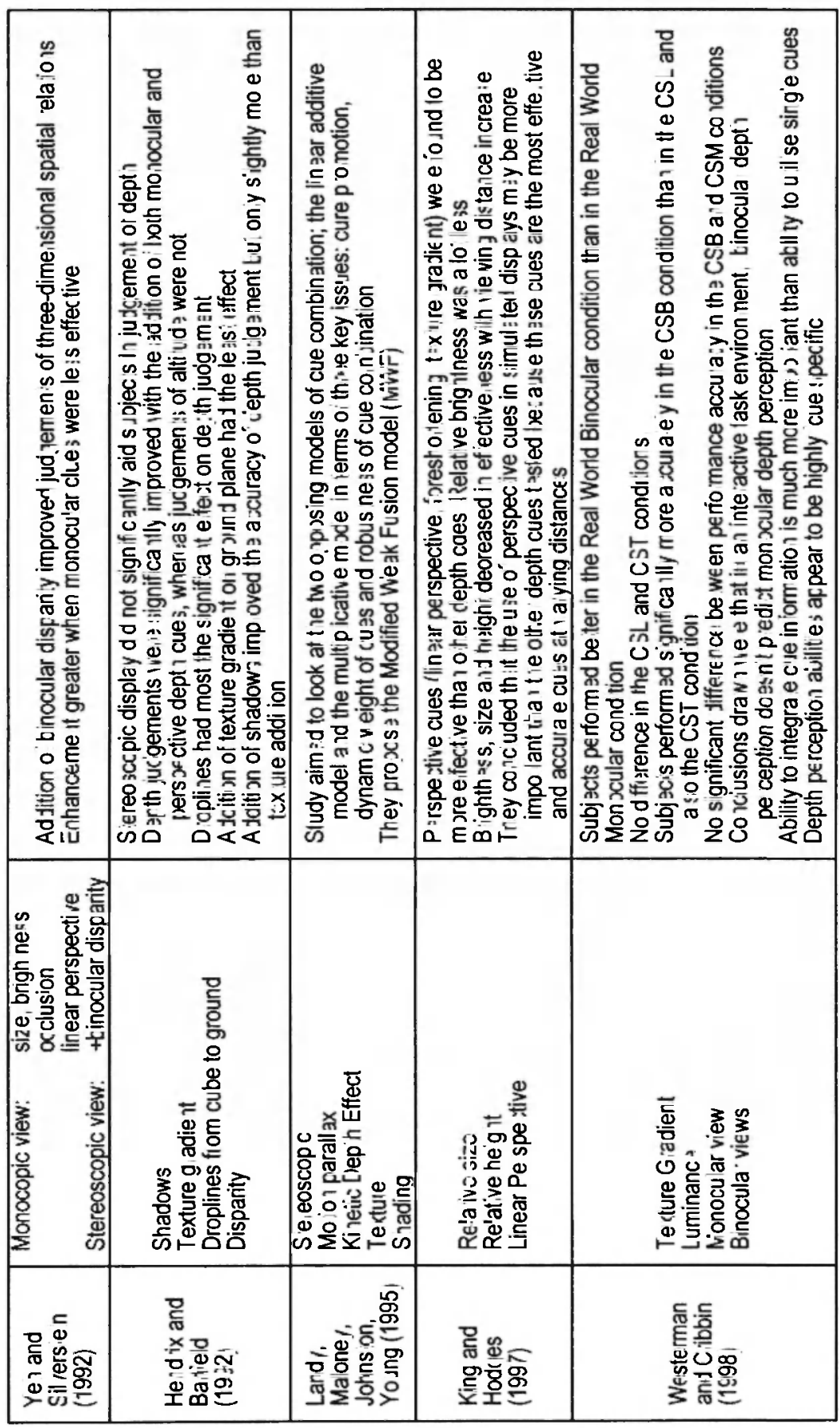


Observer (Landy et al., 1995), the Linear Combination model (Dosher et al., 1986), minimodularity (Bruno \& Cutting, 1988), and the Fuzzy Logical Model of Perception (Massaro, 1988). These models are either modular or multiplicative or, in some cases, combinations of the two contrasting approaches.

\section{APPROACHES FOR CUE COMBINATION}

The visual cue combination theories will now be explored in an attempt to understand how individuals perceive the combinations of visual depth cues that are used to present the dimensionality within desktop virtual environments. $V$ isual cue combination theories form a continuum of approaches, ranging from modular at one end to multiplicative at the other, as shown in Fig. 1. The section begins by discussing the modular approach, which suggests that individuals combine the perceptions of visual cues in a linear manner and then progresses to consider the contrasting multiplicative approach suggesting that individuals merge all the cue types together. This is followed by a discussion of various other approaches that combine the modular and multiplicative approaches, which propose to be the more realistic reflection of the manner by which individual combine visual depth cues.

\section{Modular Approach}

The Weak Observer is a model of cue combination involving the consideration of each depth cue within a scene individually. Depths are first estimated from each depth cue, which are known as 'depth maps'. Then the estimates are averaged for each depth cue, which provides the overall depth map for the scene. This is a modular approach, as the depth maps are computed independently and the rule of combination (averaging) is simple. The advantage of this model is that it is a modular structure, and therefore, considering each depth cue in isolation is easier. A problem with this model is that each depth cue provides qualitatively different information. For instance, depth given by motion parallax could be estimated in meters and depth given by texture is relative (Landy et al., 1995). To overcome this problem, we must 


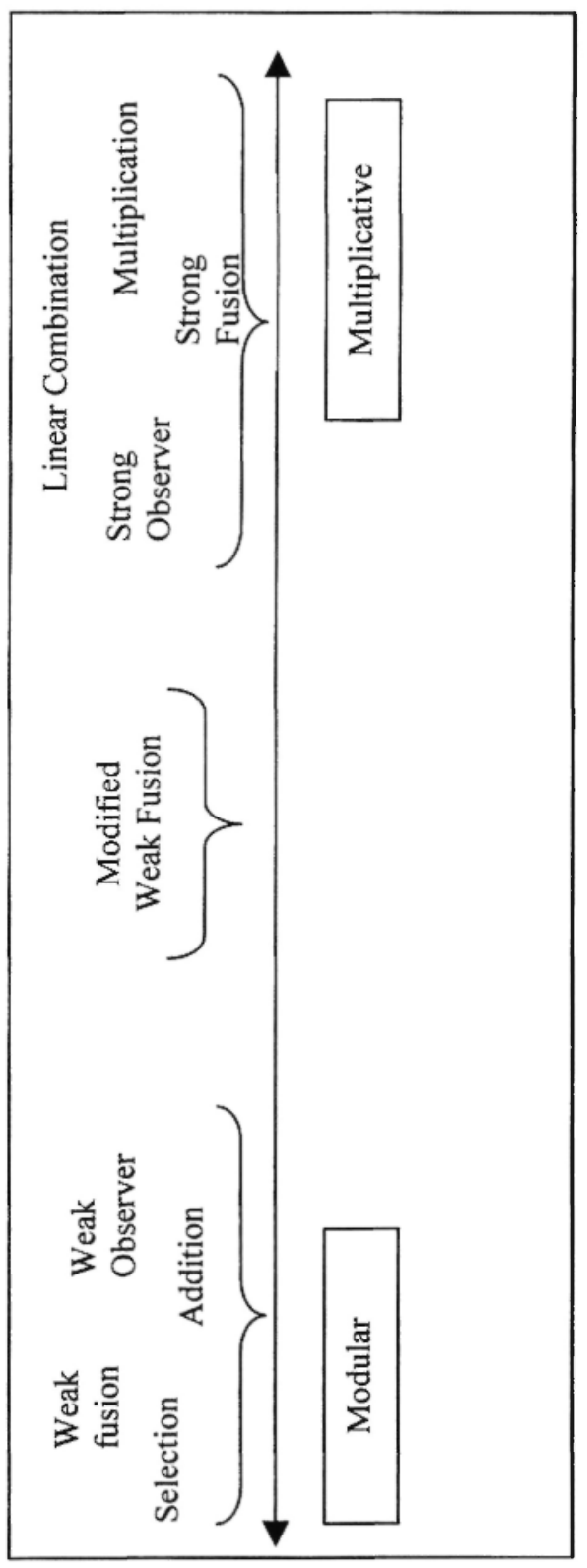

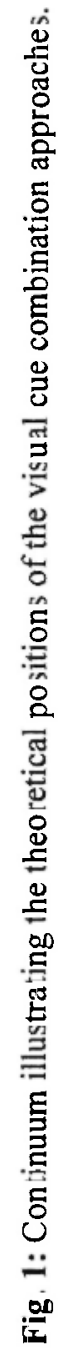


change the estimates into common units before averaging the depth maps. This process is known as 'promotion'. But even then, the possibility may exist that interpretations could be misleading. Landy et al. (1995) for example describe a situation whereby a reflection of texture may be considered the same as a textured surface. Therefore Landy et al. state that the cue combination process should be more robust and dynamic, integrating the approaches, to support the changing instances within a particular scene.

Bruno and Cutting (1988) proposed three strategies for cue combinationselection, addition, and multiplication - and called it minimodularity. Selection simply referred to the individual's ability to select the most effective cue and disregard the rest. This particular strategy matches the notion of invariant pickup, promoted by Gibson (1979). 'Addition' referred to the modular approach, reflecting the idea that an individual processes all the information sources, weighs them, and then uses the sum of the results. This is considered the most efficient cue combination model. The study by Dosher et al. (1986) also corresponds to this approach of combination, namely the Linear Combination Model. The third strategy, called Multiplication, is described by Bruno and Cutting (1988) as providing the ability for individuals to use certain visual cues to correct information from other visual cues.

\section{Multiplicative Approach}

Landy et al. (1995) describe the contrasting Strong Observer model as not modular. Nakayama and Shimojo (1992) proposed that in this model, the observer makes the most likely interpretation of the scene and this is not necessarily modular. Landy et al. draw a parallel between this model and the approaches called 'weak fusion' and 'strong fusion' put forward by Clark and Yuille (1990). With weak fusion, each depth cue is considered independently, and the estimates are combined together. With strong fusion, the combination rule is not necessarily linear, and the depth modules may act together to illustrate depth within a scene. The strong fusion model can be complex and therefore harder to test. Landy et al. describe the two ends of the cue combination spectrum, whereby on the weak side the approaches tend to be modular and on the strong side, the models and approaches tend to merge the cue types together, as illustrated in Fig. 1. It is then difficult to distinguish the 
individual depth cues and their relative effects. Westerman and Cribbin (1998) describe the 'weak observer' as a modular additive model and the 'strong observer' as a multiplicative model. Landy et al. acknowledge the weakness of the two contrasting models and maintains that a more appropriate approach in describing the way in which individuals perceive the combinations of visual depth cues would be to integrate both models and yet ensure that the fusion was as modular as possible.

\section{Other Approaches}

The Modified Weak Fusion model (MWF) is a model proposed by Landy et al. (1995) that attempts to overcome the difficulties of the two previous models. The MWF model is as modular as possible and comprises certain statistical features that attempt to account for and to reflect the potential irregularities when perceiving combinations of visual cues.

- The first feature enables different depth cues to be estimated in the same units-a process called 'promotion'.

- The next consideration is robustness, which acknowledges that statistical calculations may not be certain and provides techniques that handle the possibilities of outliers.

The next issues considered within the MWF model are reliability, ancillaries, and availability. Availability refers to the number of cues available in a natural scene. Landy et al. (1995, 396) explain,

\footnotetext{
"an estimate of cue variance should be based on the objective 'availability' of cues in the scene and on the quality of depth information available from the cue. For example, if a region of a scene contains no texture elements or if the texture elements are sparse, then the weight assigned to texture elements in that region of the scene should drop if more reliable cues are available."
}

Reliability is explained by considering the reducing influence of stereo disparity as viewing distances become larger. In these instances, the stereo cue becomes less reliable and thus should be given less weight. 
The modular and multiplicative approaches, although distinct in their processes, can be combined to some degree to produce subadditive approaches. This particular approach has been used to describe certain cue combinations more effectively and realistically (Massaro, 1988; Bruno \& Cutting, 1988; Landy et al., 1995). Yet, research is still ongoing to identify the approaches that individuals use to perceive visual cues-whether a simple multiplication approach or a combination of multiplication and addition (Bruno \& Cutting; 1988). The implications of this particular research would have a bearirg on the manner by which visual cues are implemented and perceived within the desktop virtual environment context.

The cue combination models described above highlight the ability for the modular and multiplicative approaches to integrate in order to describe a more realistic manner by which individuals perceive combinations of visual cues. Such integration has also highlighted the complexity of visual cue combination, especially with the idea that certain visual cues may conflict.

\section{VISUAL CUE CONFLICT}

When perceiving the combinations of visual depth cues used to create the dimensionality within desktop virtual environments, individuals may be required to contend with visual cues that appear to conflict. Therefore, the aim of this section is to highlight the literature that has acknowledged the importance of understanding visual cue conflicts. Conflicting visual cues maybe perceived whilst interacting with desktop virtual environments, and it is the purpose of research to identify how individuals visually evaluate this ambiguity. The section begins by reviewing a study undertaken by Dosher et al. (1986) illustrating how conflicting visual cues may provide inaccurate perceptions, which is then followed by a discussion about the limitations of visual depth cues in promoting distance perception in three-dimensional presentations.

Visual cues that are either in opposition or conflict when they are visually perceived were examined by Dosher et al. (1986). The investigators examined the two-dimensional polar projection of a three-dimensional wire cube. When the wire cube rotated clockwise, it appeared as a rigid object and when the wire cube rotated anti-clockwise, it appeared as a non-rigid object. Dosher et 
al. examined a condition in which stereo rotation disparity favored the nonrigid percept, and proximity-luminance covariance favored the rigid percept. Dosher et al. maintained that if each cue were varied independently, then this variation would change the probability of viewing rigid and non-rigid percept respectively. Their results indicated that both the stereo rotation disparity and the proximity luminance covariance, whether in combination or in conflict, show a form and motion percept. The authors also found that stereo was a stronger cue than proximity luminance covariance. The implications of this study reflected on understanding the way that people combine visual cues.

Visually perceiving cue conflict within the real world can be regarded as visually perceiving an illusion (see Gregory [1998] for extensive research in this area). Desktop virtual environments incorporate multiple visual cues to generate perceptions. The issue arises, however, as to the accuracy of these displays when attempting to perceive depth within the virtual environment. Foley (1991) concludes that distance perception is generally inaccurate, even with the visual use of multiple cues. The author postulates that individuals can perform distance judgments accurately, even with inaccurate distance perceptions, by learning from feedback. He acknowledges the difficulty in creating accurate threedimensional displays, due not only to the limited knowledge of the visual cues but also to coping with an individual's inherent ability to make rapid compensations for erroneous visual information (Foley, 1991).

The importance of understanding the effective use of visual depth cues in displaying desktop virtual environments has been illustrated through the discussions of cue combinations and conflicts. That the manner by which visual depth cues are combined can be a complex process has been acknowledged, and this complexity is further emphasized by the cognitive differences of individuals. By the perceptual theories considered so far, it is apparent that frequently generalizations have to be made about how individuals visually perceive certain attributes. Although such generalizations have attempted to provide insights to the perceptual process that are applied to certain contexts, such as desktop virtual environments, the insights can be considered limited with respect to the effects of individual differences (Chen et al., 2000). Therefore, in the following section we will explore the issue of individual differences to highlight prospective areas of interest in the desktop virtual environment context. 


\section{INDIVIDUAL DIFFERENCES IN THE USE OF VISUAL CUES}

To emphasize the need for research to consider individual differences for the perception of visual depth cues, this section begins by presenting a brief overview of the various domains in the area and identifies the visual perception domain as an appropriate approach. This domain consists of various factors that are listed in Table 2 and then briefly described with respect to their applicability to the visual depth cues and to the desktop virtual environment context.

Desktop virtual environment displays still endure certain interactive limitations that seem to correspond to the individual's perceptual ability (Stanney et al., 1998). It has therefore been proposed that attention to individual differences on the use of depth cues would benefit the display of desktop virtual environments (Westerman \& Cribbin, 1998). Current research has considered individual differences in the use of binocular and monocular depth cues when perceiving desktop virtual environments, and the results indicate that individual differences in depth perception of desktop virtual environments is strongly dependent on visual depth cues (Westerman \& Cribbin, 1998).

The topic of individual differences is an established research area. The literature has acknowledged the extent to which it has developed and now proposes that it be applied to the broad area of virtual environments (Carroll, 1993; Dillon \& Watson, 1996; Chen et al., 2000). Individual differences consist of various domains, including reasoning, memory and learning, cognitive speed, and visual perception (Carroll, 1993). It is the visual perception domain that provides an approach on which to assess an individual's ability to visually perceive visual depth cues. Such factors as visualization (VZ), spatial relations (SR), perceptual speed (S), length estimation (LE), and illusion (IL) are particularly applicable (Carroll, 1993). Table 2 illustrates these factors, the examined ability, and the types of tests involved, which are based on the 1963 ETS factor kit and other studies (French et al., 1963; Eliot \& Smith 1983). For more detail and further descriptions of the tests listed in Table 2, see Carroll (1993).

A brief discussion about the various factors will now follow to illustrate their relevance to the desktop virtual environment context. Table 2 lists 
Table 2

Overview of visual depth cue studies

\begin{tabular}{|c|c|c|}
\hline Factors & Ability & Tasks/Tests \\
\hline $\begin{array}{l}\text { Visualisation } \\
\text { (VZ) }\end{array}$ & $\begin{array}{l}\text { to comprehend } \\
\text { movements in } 3 \mathrm{D} \\
\text { space }\end{array}$ & $\begin{array}{l}\text { 1. Paper Formboard and } \\
\text { Assembly Tasks } \\
\text { 2. Block Tasks } \\
\text { 3. Block Rotation Tasks } \\
\text { 4. Paper Folding Tasks } \\
\text { 5. Surface Development Tasks } \\
\text { 6. Perspective Tasks }\end{array}$ \\
\hline $\begin{array}{l}\text { Spatial } \\
\text { Relation } \\
(\mathrm{SR})\end{array}$ & $\begin{array}{l}\text { to perceive spatial } \\
\text { patterns or to } \\
\text { perceive object } \\
\text { orientation in 3D } \\
\text { space }\end{array}$ & $\begin{array}{l}\text { 1. Card Rotation Test } \\
\text { 2. Cube Comparisons Tests } \\
\text { 3. Spatial Orientation Test }\end{array}$ \\
\hline $\begin{array}{l}\text { Perceptual } \\
\text { Speed (P) }\end{array}$ & $\begin{array}{l}\text { in speed, to make } \\
\text { comparisons, } \\
\text { identify figures } \\
\text { and conduct } \\
\text { simple visual } \\
\text { search tasks }\end{array}$ & $\begin{array}{l}\text { 1. Finding A's Test } \\
\text { 2. Number Comparison Test } \\
\text { 3. Identical Picture Test }\end{array}$ \\
\hline $\begin{array}{l}\text { Length } \\
\text { Estimation } \\
\text { (LE) }\end{array}$ & $\begin{array}{l}\text { to compare lengths } \\
\text { of lines and } \\
\text { distances, on paper }\end{array}$ & $\begin{array}{l}\text { 1. Estimation of Length Test } \\
\text { 2. Shortest Road Test } \\
\text { 3. Nearer Point Test }\end{array}$ \\
\hline Illusion (IL) & $\begin{array}{l}\text { to perceive or } \\
\text { misperceive visual } \\
\text { illusions }\end{array}$ & $\begin{array}{l}\text { 1. Shape and direction illusion } \\
\text { 2. Size contrast illusion } \\
\text { 3. Overestimation illusion } \\
\text { 4. Underestimation illusion }\end{array}$ \\
\hline
\end{tabular}

visualization as a factor that attempts to assess an individual's ability to comprehend and manipulate objects within three-dimensional spaces, which is particularly applicable to perceiving visual depth cues because they are fundamental to the presentation of the third dimension in desktop virtual environments. Spatial relations considers the individual's ability to perceive the orientations of objects in the third dimension and the ability to perceive spatial patterns. The three tasks supporting this factor involve judging the orientation, rotation, and comparison of cubes, which is directly applicable to 
the ability of individuals to judge depth in different orientations within desktop virtual environments (Carroll, 1993).

Perceptual speed is a factor that judges the individual's speed in identifying the effects of the visual depth cues on the perception of depth within desktop virtual environments. Length estimation is a vital factor that assesses the individual's ability to judge spatial distances. The illusion factor directly relates to the use of visual depth cues that combine to create the illusion of depth and dimensionality for desktop virtual environments. The tests for this factor consider shape, direction, and size irregularities and assess the extent to which this affects individuals when perceiving depth in desktop virtual environments (Carroll, 1993).

Individual differences has been broadly considered in terms of the visual perception domain. Various factors have been identified as being suitable for assessing the extent to which individuals abilities effect their perception of visual depth cues in desktop virtual environments. Egan (1988) has stressed that domain-specific knowledge would only begin to determine performance after individuals have gained experience with perceiving the dimensionality that is presented in a desktop virtual environment. Investigating individual differences on perceiving visual depth cues would enable implications to be drawn for the minimum and maximum display requirements for presenting conducive desktop virtual environment and supporting effective visual perception.

\section{RESEARCH AGENDA}

The review of visual perception issues within this paper has highlighted the prospective areas of research within the desktop virtual environment context. The various issues that have been discussed in the paper include assessing the significant use of the desktop virtual environment tool within several commercial industries. A review was presented of generic visual perception theories that have been formulated according to nativist and empiricist viewpoints, which illustrates the complexity of understanding visual perception within the real world and questions the direct applicability of the theories to desktop virtual environments. We also explored the implications of existing studies that have examined visual cue combination 
and visual cue conflict theories, which have acknowledged necessity for exploring the theories in the context of desktop virtual environments. Finally, in this review, we broadly considered the growing importance of individual differences for visual cue usage within the desktop virtual environment context. The literature review of these areas not only emphasized further investigative areas but also provided a theoretical foundation for building on existing studies in the area (see Surdick et al., 1997; Westerman \& Cribbin, 1998). From this position, a research agenda can be formulated to define the specific research areas that require attention. Figure 2 illustrates the research agenda in terms of the three issues that have been identified as important from this review. These issues will be broadly discussed in terms of their contribution to understanding the effects of visual depth cues when perceiving desktop virtual environments.

The research agenda identifies three relevant areas that contribute to furthering the current understanding of perceiving desktop virtual environments. The areas were determined after broadly reviewing various issues concentrating on visual depth cues and their effects on visually perceiving depth in the virtual environment. The complexity of perceiving visual depth cues has been highlighted through the review of the different perceptual approaches and by acknowledging that integrating them may more accurately represent visual perception. Therefore, to contribute to the further understanding of the effects of visual depth cues on the perception of desktop virtual environments, the research agenda proposes to establish an overview of visual depth cue usage and the cue combination theories. The agenda then progresses to investigate the effects of cue conflict or illusions on desktop virtual environments, and finally the agenda emphasizes the need to involve factors of individual differences when investigating visual perception of desktop virtual environments. These three issues are briefly discussed in the following sections.

\section{Overview of Visual Depth Cues}

This area of the research involves considering visual depth cues in isolation and identifying the individual strengths of each and the effects of 


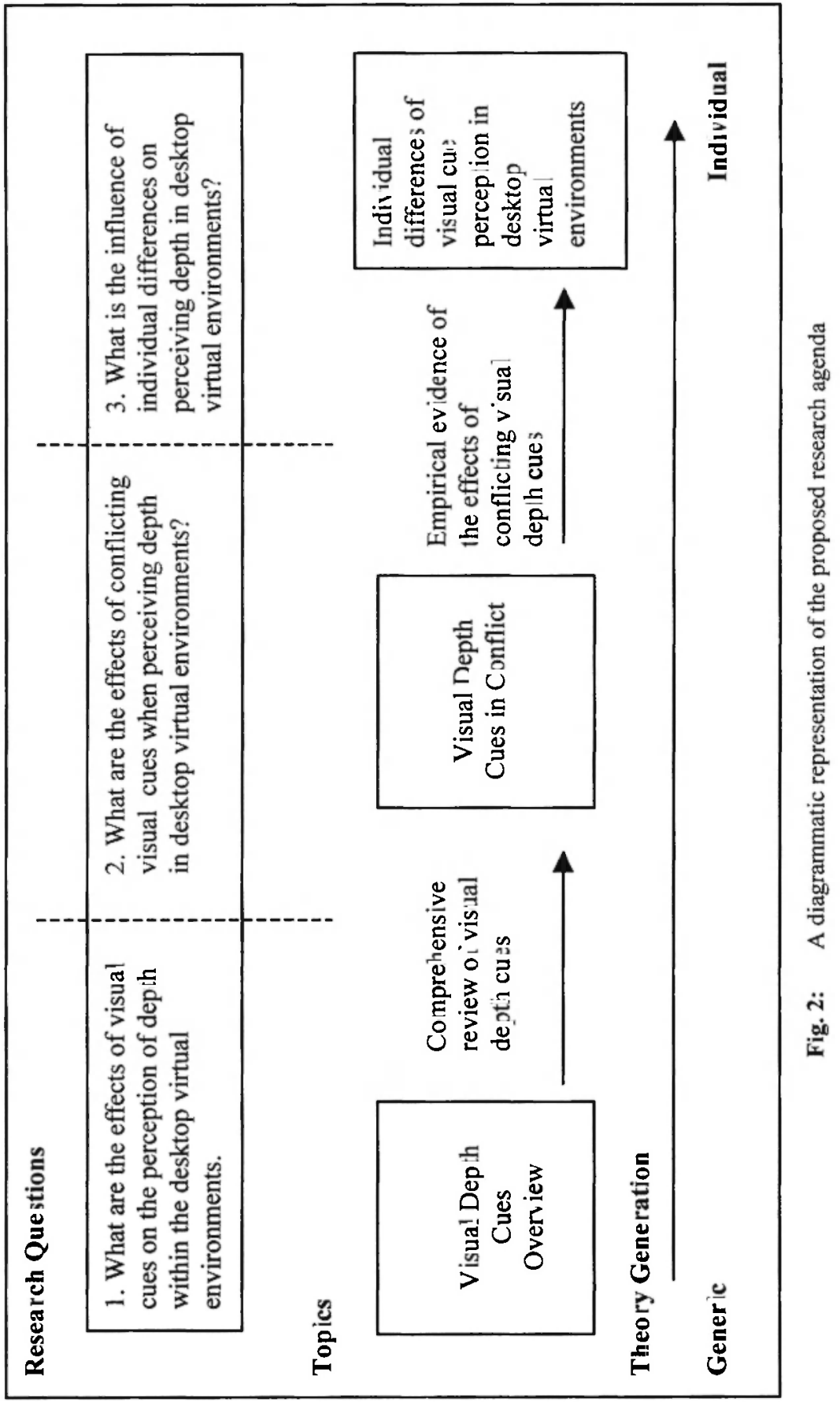


specific combinations when visually perceiving the desktop virtual presentations. The empirical approach enables visual cues to be isolated for investigating their individual effects on visual depth perception. The experimental work would be conducted to investigate the hypothesis that when perceiving desktop virtual environments, firstly motion is the most dominant visual cue (Wickens et al., 1989, Surdick et al., 1997), and secondly, visual cue combinations are predominantly perceived in a modular, additive approach (Bruno \& Cutting, 1988).

The objective is to provide a comprehensive study of the visual depth cues to strengthen desktop virtual environment aspects of visual depth cue use and broadly consider the results in respect to the cue combination theories. Figure 2 illustrates that this comprehensive review would be drawn from studies based upon generic visual perception theories involving approaches that when combined may reflect the perception of visual depth cues more accurately. This approach illustrates the complexity of identifying the effects of visual depth cues on visually perceiving desktop virtual environments.

\section{Visual Cue Conflict}

This area of the research examines visual cue conflict, which refers to the ability of certain visual cues to provide conflicting depth information about a particular desktop virtual environment display. This concept is important to understand, especially as desktop virtual environments are interactive and dynamic in nature and are often used to replicate real world situations, such as for training applications in the aviation industry. Experimental work would investigate the hypothesis that when certain visual cues are in conflict whilst perceiving desktop virtual environments, the most dominant cue of the ambiguous combination takes precedence. As Fig. 2 illustrates, the result of this topic is the provision of more empirical evidence about the way in which conflicting cues are visually perceived in desktop virtual environments, as currently little work is being done in this area. This evidence leads on to introducing the growing importance of considering the effects of individual differences for perceiving visual depth cues within desktop virtual environments. 


\section{Individual Differences in the Use of Visual Cues}

The final stage of the research extends the visual cue review to consider individual differences in visual cue use when perceiving desktop virtual environments, as illustrated in Fig. 1. The term 'individual differences' refers to the influences of the individual's cognitive abilities to task performance. This topic has been covered quite extensively as a specific research field (Dillon \& Watson, 1996, Chen et al., 2000) and can be applied to the area of visual depth cues to answer questions about the types of visual cues that individuals employ to perceive desktop virtual environments.

The visual perception domain of individual differences provides an approach to considering the influences of visual depth cues. This domain involves various factors, including visualization, spatial relations, perceptual speed, length estimation, and illusions (Carroll, 1993). Each factor involves completing specific tasks to provide an assessment or judge the individual's ability to comprehend three-dimensional spatial environments with respect to orientation, sense of depth, and speed of perceptual processing (Egan, 1988; Carroll, 1993). The research question for this particular issue investigates the role of individual differences when perceiving visual depth cue. The underlying approaches enable visual cue theories to acknowledge the individual's cognitive and perceptual abilities.

The research agenda has been established as an initial framework for proposing and highlighting the importance of research for the area of visual depth cues. The three issues aim to provide insightful contributions to understanding visual perception of the depth cues that are presented in desktop virtual environments and, as the research proceeds, to generate an appropriate theory concerned with visual perception and individual differences with respect to desktop virtual environments.

\section{SUMMARY}

This paper has provided an extensive review of visual perception issues for perceiving depth cues in desktop virtual environments. The aim of the paper was to establish an agenda for research that will contribute new 
findings to the field from basic empirical investigations. The issues that have been considered within the paper began with an overview of the various industrial areas that employ the desktop virtual environment tool. This synopsis emphasized the popularity of virtual tools and illustrated their importance for this research. The paper proceeded to consider broadly the cognitive area of visual perception in terms of the various theories of perception. It was important to consider the contrasting approaches in terms of understanding how visual depth cues may be perceived. The paper then proceeded to discuss the various issues relating to perceiving visual depth cues - such as cue combination and conflict and the effects and influences of individual differences-in the light of expanding current knowledge about visual perception of desktop virtual environments.

The research agenda highlighted three issues:

1. presenting a comprehensive review of the effects of visual depth cues,

2. considering the effects of conflicting visual cues, and then

3. identifying the roles of individual differences on visual cue perception for the context of desktop virtual environments.

The research agenda has been proposed as an initial framework for proposing and highlighting the importance of research for the area of visual depth cues and for scoping research activities to contribute to the development of this challenging and topical area.

\section{REFERENCES}

Aleksandric, D. and Duboka, C. 1997. Virtual reality-New technology for systems engineering, 16th International Conference Science and Motor Vehicles '97, Belgrade, Yugoslavia

Bruno, N. and Cutting, J.E. 1988. Minimodularity and the perception of layout, Journal of Experimental Psychology: General, 117, 161-170.

Carroll, J.B. 1993. Human Cognitive Abilities. A Survey of Factor-Analytic Studies.

Chen, C. Czerwinski, M. and Macredie, R. 2000. Individual differences in virtual environments-Introduction and overview. Journal of the American Society for Information Science, 51, 499-507. 
Clark, J.J. and Yuille, A.L. 1990. Data fusion for sensory information processing systems, Kluwer, Boston, Massachusetts, USA.

Csikszentmihalyi, M. and Kubey, R. 1981. Television and the rest of life: A systematic comparison of subjective experience, Public Opinion Quarterly, 45, 317-328.

Cutting, J.E. 1986. Perception with an eye for motion, MIT Press, Cambridge, Massachusetts, USA.

Deitz, D. 1995. Real engineering in a virtual world, Mechanical Engineering, $117,78-85$.

Dillon, A. and Watson, C. 1996. User analysis in $\mathrm{HCl}$-The historical lessons from individual differences research, International Journal of HumanComputer Studies, 45, 619-637.

Dosher, A.D. Sperling, G. and Wurst, S.A. 1986. Tradeoffs between stereopsis and Proximity Luminance Covariance as determinants of perceived 3D structure, Vision Research, 26, 973-990.

Egan, D.E. 1988. Individual differences in human-computer interaction, in: Handbook of human-computer interfaces, edited by Helander, M. Landauer, T.K. and Prabhu, P, Elsevier Science, New York, NY, USA, 543-565.

Ellis, S.R. Begault, D.R and Wenzel, E.M. 1997. Virtual environments as human computer interfaces in: in: Handbook of human-computer interfaces, edited by Helander, M. Landauer, T.K. and Prabhu, P, Elsevier Science, New York, NY, USA, 163-201.

Eliot, J. and Smith, I.M. 1983. An International directory of spatial tests, Windsor, England: NFER/Nelson and Atlantic Highlands, Humanities Press, New Jersey, USA.

EPSRC. 2000. Human factors of virtual reality, Report for Workshop held $21^{\text {st }} / 22^{\text {nd }}$ February, Manchester, UK.

Foley, J.M. 1991. Stereoscopic distance perception, in: Pictorial communication in virtual and real environments, edited by Ellis, S.R., Taylor \& Francis, Bristol, Pennsylvania, USA.

French, J.W., Ekstrom, R.B. and Price, L.A. 1963, Manual and kit of reference tests for cognitive factors, Educational Testing Service, Princeton, New Jersey, USA.

Gibson, J.J. 1950. The Perception of the visual world, The Riverside Press, Riverside, California, USA. 
Gibson, J.J. 1968, The senses considered as perceptual systems, Allen and Unwin, London, UK.

Gibson, J.J 1979, The ecological approach to visual perception, Houghton Mifflin, London, UK.

Gordon, I.E. 1989. Theories of visual perception, Wiley, Chichester, UK.

Gregory, R. 1998, Eye and brain: The psychology of seeing, Oxford University Press, Oxford, UK.

Hebb, D.O. 1949. The organization of behavior, John Wiley, New York, NY, USA.

Hendrix, C. and Barfield, W. 1995. Relationship between monocular and binocular depth cues for judgements of spatial information and spatial instrument design, Displays, 16, 103-113.

Hubbold, R., Murta, A, West, A, and Howard, T. 1993 Design issues for virtual reality systems, Ist Eurographics Workshop on Virtual Environments. Barcelona, Spain.

Koffka, K .1935. Principles of Gestalt psychology, Kegan Paul, Trench, London, UK.

Landy, M.S., Maloney, L.T., Johnston, E.B. and Young, M. 1995 Measurement and modeling of depth cue combination: in defense of weak fusion, Vision Research, 35, 389-412.

Marr D. 1982. Vision: A computational investigation into the human representation and processing of visual information, W.H. Freeman, San Francisco, California, USA.

Massaro, D.W. 1988, Ambiguity in perception and experimentation, Journal of Experimental Psychology: General, 117, 417-421.

McConathy, Doyle. 1991. Interactive displays in medical art, in Pictorial communication in virtual and real environments, edited by Ellis, S.R., Taylor \& Francis, Bristol, Pennsylvania, USA.

Nagata, S. 1991. How to reinforce perception of depth in single twodimensional pictures, in Pictorial communication in virtual and real environments, edited by Ellis, S.R., Taylor \& Francis, Bristol, Pennsylvania, USA.

Nakayama, K. and Shimojo, S. 1992, Experiencing and perceiving visual surfaces, Science, 257, $1357-1363$ 
Norman, D. 1988. The psychology of everyday things. Basic Books, Inc., New York, NY, USA.

Stanney, K.M., Mourant, R.R and Kennedy, R.S. 1998. Human factors issues in virtual environments: A review of the literature, Presence, 7, 327-3510.

Surdick, R.T., Davis, E.T. and Hodges, L.F. 1997, The perception of distance in simulated visual displays: A comparison of the effectiveness and accuracy of multiple depth cues across viewing distances, Presence, 6, 513-531.

Wann, J. and Mon-Williams, M. 1996. What does virtual reality need?: Human factors issues in the design of three-dimensional computer environments. International Journal of Human-Computer Studies. 44, 829-847.

Westerman, S.J. and Cribbin, T. 1998. Individual differences in the use of depth cues: implications for computer- and video-based tasks, Acta Psychologica 99, 293-310.

Weintraub D.J. and Walker. E.L. 1973. Perception, Brooks/Cole, Belmont, California, USA.

Wickens, C.D., Todd, S., and Seidler, K. 1989. Three-dimensional displays: Perception, implementation and applications. CSERIAC. 